\title{
Opioid Taper Is Associated with Subsequent Termination of Care: a Retrospective Cohort Study
}

\author{
Hector R. Perez, MD, MS ${ }^{7}$, Michele Buonora, $M D, M S^{l}$, \\ Chinazo O. Cunningham, MD, MS', Moonseong Heo, $\mathrm{PhD}^{2}$, and \\ Joanna L. Starrels, MD, MS ${ }^{7}$
}

${ }^{1}$ Montefiore Medical Center, Albert Einstein College of Medicine, Bronx, NY, USA; ${ }^{2}$ Department of Public Health Sciences, College of Behavioral, Social and Health Sciences, Clemson University, Clemson, SC, USA.

BACKGROUND: Opioid tapering is increasingly utilized by providers to decrease risks of chronic opioid therapy, but it is unknown whether tapering is associated with termination of care.

OBJECTIVE: To determine whether patients taking chronic opioid therapy who experienced opioid tapers were at greater risk of subsequently terminating their care compared with those who were continued on their doses. DESIGN: Retrospective cohort study of patients in a large, urban health system between 2008 and 2012 with 2 years of follow-up.

PARTICIPANTS: Adult patients prescribed a stable baseline dose of chronic opioid therapy of at least 25 morphine milligram equivalents per day during a baseline year.

MAIN MEASURES: An opioid taper during an exposure year, defined as a reduction in the average daily dose of at least 30\% from the baseline dose in both of the two 6month periods in the year following the baseline year. Opioid dose continuation was defined as any increase in dose, no change in dose, or any decrease up to $30 \%$ compared with baseline dose in the exposure year. The primary outcome was termination of care, defined as no outpatient encounters in the health system, in the year following the exposure year.

KEY RESULTS: Of 1624 patients on chronic opioid therapy, 207 (15.5\%) experienced an opioid taper and 78 $(4.8 \%)$ experienced termination of care. Compared with opioid dose continuation, opioid taper was significantly associated with termination of care (AOR 4.3 [95\% CI 2.2-8.5]).

CONCLUSIONS: Opioid taper is associated with subsequent termination of care. These findings invite caution and demonstrate the need to fully understand the risks and benefits of opioid tapers.

KEY WORDS: chronic pain; primary care; patient safety; care management.

J Gen Intern Med 35(1):36=42

DOI: $10.1007 / \mathrm{s} 11606-019-05227-9$

(c) Society of General Internal Medicine 2019

Received August 8, 2018

Revised April 3, 2019

Accepted June 18, 2019

Published online August 19, 2019

\section{INTRODUCTION}

Opioid tapering is a recommended strategy employed to reduce risks associated with chronic opioid therapy (COT) by reducing doses to a lower level. ${ }^{1}$ Though the decision to taper is an individualized one, many guidelines recommend tapering for patients on COT at the highest risk of misuse and overdose. $^{2,3}$ Though the incidence of opioid tapering is unknown, clinical experience and anecdotal evidence suggest that its practice has become widespread. ${ }^{4,5}$

Nonetheless, opioid tapering can lead to unintended negative consequences. Termination of care, when patients leave their providers or drop out of care entirely, is a common negative consequence with clinical significance. Patient conflict, which can often lead to termination of care, is the most common reason why providers have concerns about initiating tapers. $^{6-9}$ Termination of care can place patients on COT at risk for opioid misuse and illicit opioid use, either because of poorly controlled pain or because of self-treatment of opioid withdrawal. Additionally, for patients with comorbid chronic conditions, termination of care can increase risk for disease progression and other health complications. ${ }^{10-13}$

In this study of patients on COT in a large urban medical center, we sought to determine whether an opioid taper was associated with subsequent termination of care. We hypothesized that patients on COT who had opioid tapers would have greater odds of terminating their care compared with patients on COT who continued their doses.

\section{METHODS}

\section{Overview and Setting}

We performed a retrospective cohort study of patients on COT for chronic non-cancer pain between July 2008 and December 2014 within a large, urban academic healthcare system located in the Bronx, NY. Using electronic medical record (EMR) data, we identified a cohort of patients prescribed a stable dose of COT for at least a year (the "baseline year"), determined whether or not they had an opioid taper in the following year (the "exposure year"), and then determined if they terminated their care in the subsequent year (the "outcome year;" see 


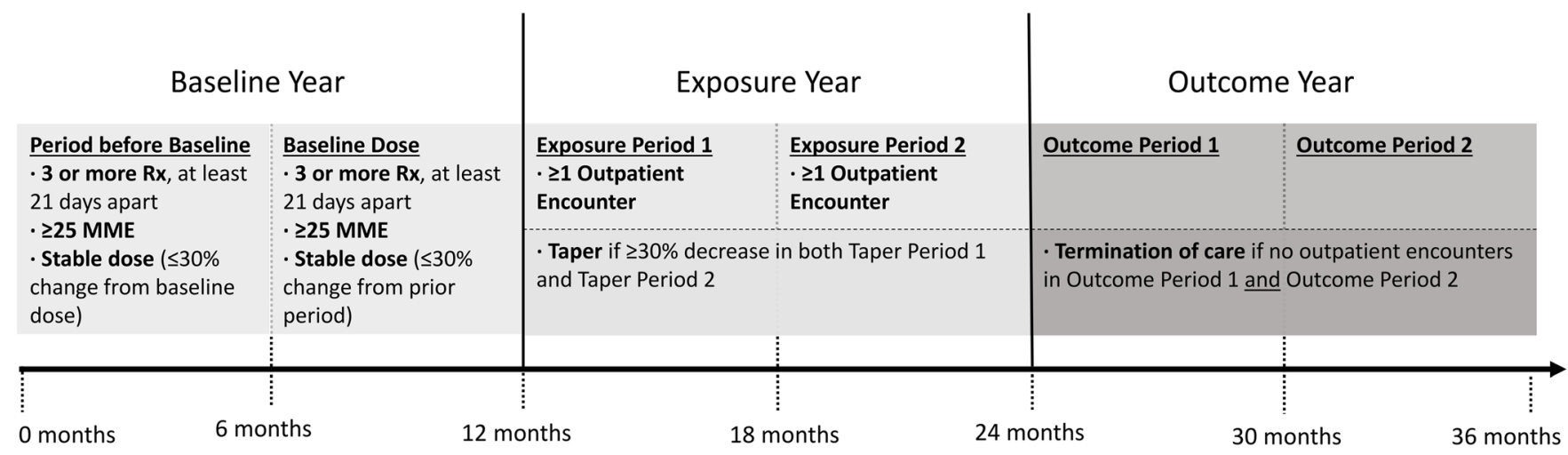

Figure 1 Defining the baseline year, the exposure year, and the outcome year.

Fig. 1). The study was approved by the Albert Einstein College of Medicine and Montefiore Medical Center Institutional Review Board.

\section{EMR Prescription Data}

To identify the cohort and determine the opioid dose for each patient, opioid prescription data were extracted from the health system's EMR. Individual opioid prescriptions were organized into distinct 6-month periods. Within each time period, we used standard conversion tables to determine the morphine milligram equivalents (MME) per prescription and summed these to determine the total MME per period, then calculated an average daily opioid dose by dividing the total MME per period by $180 .^{14} \mathrm{We}$ defined "COT" as three or more opioid prescriptions, at least 21 days apart, in two consecutive 6-month periods during the baseline year. ${ }^{15}$ We defined "stable dose" as an average daily MME that varied by less than $30 \%$ between two consecutive 6-month periods. ${ }^{14}$

Prescriptions for all formulations of opioid medications prescribed in an outpatient setting were included except buprenorphine, liquid methadone, and intravenous/injection medications. Prescriptions that lacked information necessary to calculate the daily opioid dose (e.g., date or quantity prescribed) were excluded from dose calculations, as were outlier prescriptions where total dose exceeded 75,000 MME (together these comprised $<1 \%$ of prescriptions)

\section{Participants}

Patients were eligible for inclusion if they (1) were 18 years or older; (2) were prescribed a stable baseline dose of COT of at least $25 \mathrm{MME}$ per day during a baseline year between July 2008 and December 2012; and (3) had at least one outpatient encounter in the health system during each of the two 6month periods during the exposure year. Patients were excluded if they (1) died during the exposure year, as determined by linkage to the Social Security death index, or (2) if they had a cancer diagnosis (ICD-9 codes [140-209, 230-239]) before their baseline year.
We restricted the sample to patients with a baseline dose of at least $25 \mathrm{MME}$ because we wanted the sample to reflect patients for which a subsequent taper, defined in more detail below as a relative change from a baseline dose, would represent a meaningful change. A patient may have qualified multiple times for inclusion, but they were only included in the study cohort at the earliest time point that they met inclusion criteria.

\section{Opioid Taper Definitions}

In the exposure year, we determined if there was an opioid taper. No standard definition for an opioid taper exists, so we defined opioid taper in two separate ways. ${ }^{16}$ The definition of opioid taper we utilized for our primary analysis was a reduction in average daily MME of at least $30 \%$, compared with the baseline dose, in both of the two 6-month periods during the exposure year. For example, a patient with a baseline dose of $100 \mathrm{MME}$ was required to have a decrease to $70 \mathrm{MME}$ or lower in both 6-month periods during the exposure year. We selected a $30 \%$ reduction as our primary definition in this study because we believe it has face validity as a clinically meaningful reduction in dose. Having excluded patients with a baseline dose less than $25 \mathrm{MME}$ per day, a 30\% dose reduction is equivalent to at least 7.5 MME per day, i.e., $7.5 \mathrm{mg}$ of hydrocodone or $5 \mathrm{mg}$ of oxycodone. We desired greater specificity in identifying a taper, so we required dose reductions in two consecutive 6-month periods, which would reduce the likelihood we would misclassify a gap in care or fluctuation in opioid prescribing as a taper. We also employed a secondary definition of opioid taper in sensitivity analyses described below, consistent with definitions used in two recent clinical trials of opioid tapering. ${ }^{17,18}$ The secondary definition was a reduction in average daily MME of at least $50 \%$, compared with the baseline dose, in both of the two 6-month periods during the exposure year.

In order to examine the association of tapering with termination of care, we identified a comparison group of patients who had their doses continued. Patients with dose continuation ("continued") were defined as those who experienced any increase in dose compared with baseline, no change in dose 
compared with baseline, or at most a 30\% reduction compared with the baseline dose in both 6-month periods during the exposure year. A third group of participants who did not meet criteria for the tapered or continued groups ("unspecified") had one 6-month period of at least a 30\% reduction compared with the baseline dose in the exposure year and one 6-month period with any increase, no change, or at most a $30 \%$ reduction in dose compared with the baseline dose. Because we could not ascertain whether a change in dose represented an intent to taper that was unsustained or another kind of fluctuation in dose, these patients were not included in the primary analysis. However, they were included in sensitivity analyses described below.

\section{Primary Outcome}

The primary outcome was termination of care in the outcome year. A patient was considered to have terminated care if the patient had no outpatient visits, defined as office visits with any primary care provider or any specialist in the health system, during the outcome year.

\section{Covariates}

We extracted sociodemographic and clinical data from the EMR. Sociodemographic data included patients' age, gender, and preferred language. Patients were classified into four mutually exclusive racial/ethnic categories based on selfreported information in the electronic medical record: (1) non-Hispanic white, (2) non-Hispanic black, (3) Hispanic, and (4) other, including multiracial, or unknown. We determined the total number of outpatient visits during the exposure year as a measurement of frequency of healthcare visits and categorized ICD-9 pain diagnoses each patient had accrued before the beginning of their baseline year into 11 clinically relevant, non-mutually exclusive categories of painful conditions. ${ }^{19}$ Using ICD-9 codes before the baseline year, we also determined if patients had a diagnosis of alcohol use disorder, tobacco use disorder, non-opioid-related substance use disorder, or any mental health disorder (defined as any diagnosis of a mood disorder, anxiety disorder, schizophrenic disorder, or post-traumatic stress disorder). Opioid use disorder, as determined by ICD-9 codes, was not included as a covariate because these diagnoses are unreliable and prone to misclassification in patients on chronic opioid therapy. ${ }^{20}$ Lastly, we calculated the modified Elixhauser medical complexity score, ranging from 0 to 31 , for each patient at the beginning of their baseline year. ${ }^{21}$

\section{Statistical Analysis}

All analyses were conducted using STATA version 14.2 (StataCorp, College Station, TX). Our primary analysis examined whether an opioid taper was associated with subsequent termination of care compared with "continued" opioid use. We used multivariate logistic regression to estimate adjusted odds ratios for termination of care during the outcome year by the presence of an opioid taper during the exposure year. The "continued" group was used as the comparison group in the primary analysis as tapering versus continuing opioids is the most clinically meaningful comparison for clinicians considering a taper. Patients in the "unspecified" group were not included in the primary analysis but were included in sensitivity analyses outlined below.

Covariates, decided a priori, included patient age, gender, race and ethnicity, preferred language (English vs. non-English), baseline dose, the number of total outpatient visits during the exposure year, the Elixhauser medical complexity score, and the number of pain category diagnoses. We included the number of pain category diagnoses as a proxy for burden of pain as we did not have data on pain intensity or functioning in this retrospective study. We also controlled for temporal effects by assigning each participant's baseline year as a covariate.

We conducted additional sensitivity analyses to determine whether findings would change based on: (1) different approaches to considering the "unspecified" group, and (2) a different definition of opioid taper. First, we defined our comparison group more broadly by including those in the unspecified group along with those in the continued group as our comparators (sensitivity analysis 1). Alternatively, we defined our taper group more broadly by including those in the unspecified group in the opioid taper group (sensitivity analysis 2 ). In doing so, we aimed to produce a range of possible effect sizes based on the assumptions we made in defining the taper and comparison groups. Second, we re-ran the primary and sensitivity analyses with the $50 \%$ taper definition described in the "Opioid Taper Definitions" section.

\section{RESULTS}

A total of 1624 patients met the eligibility criteria (see Fig. 2). The majority were female $(54.3 \%)$ and had back and/or extremity pain $(63.1 \%$ and $63.3 \%$, respectively) (see Table 1$)$. The median daily baseline dose was 90 MME. Overall, 207 (12.7\%) patients had an opioid taper, 1,131 patients had dose continuation $(69.6 \%)$, and 286 were unspecified (17.6\%). Of the 207 patients tapered, 19 (9.2\%) had complete discontinuation of opioids during the exposure year. Among patients in the taper group who had a reduction without complete discontinuation, the median dose reduction during the exposure year was $71.4 \%$, while patients in the continued group had a median dose increase of $9.1 \%$.

During the outcome year, 78 patients $(4.8 \%)$ experienced termination of care, including 18 (8.7\%) who had experienced a taper, $22(2.0 \%)$ who had been continued, and 38 (13.3\%) who were unspecified. In our primary analysis comparing patients with opioid tapers versus those continued on opioids, opioid taper was associated with greater than four times the 


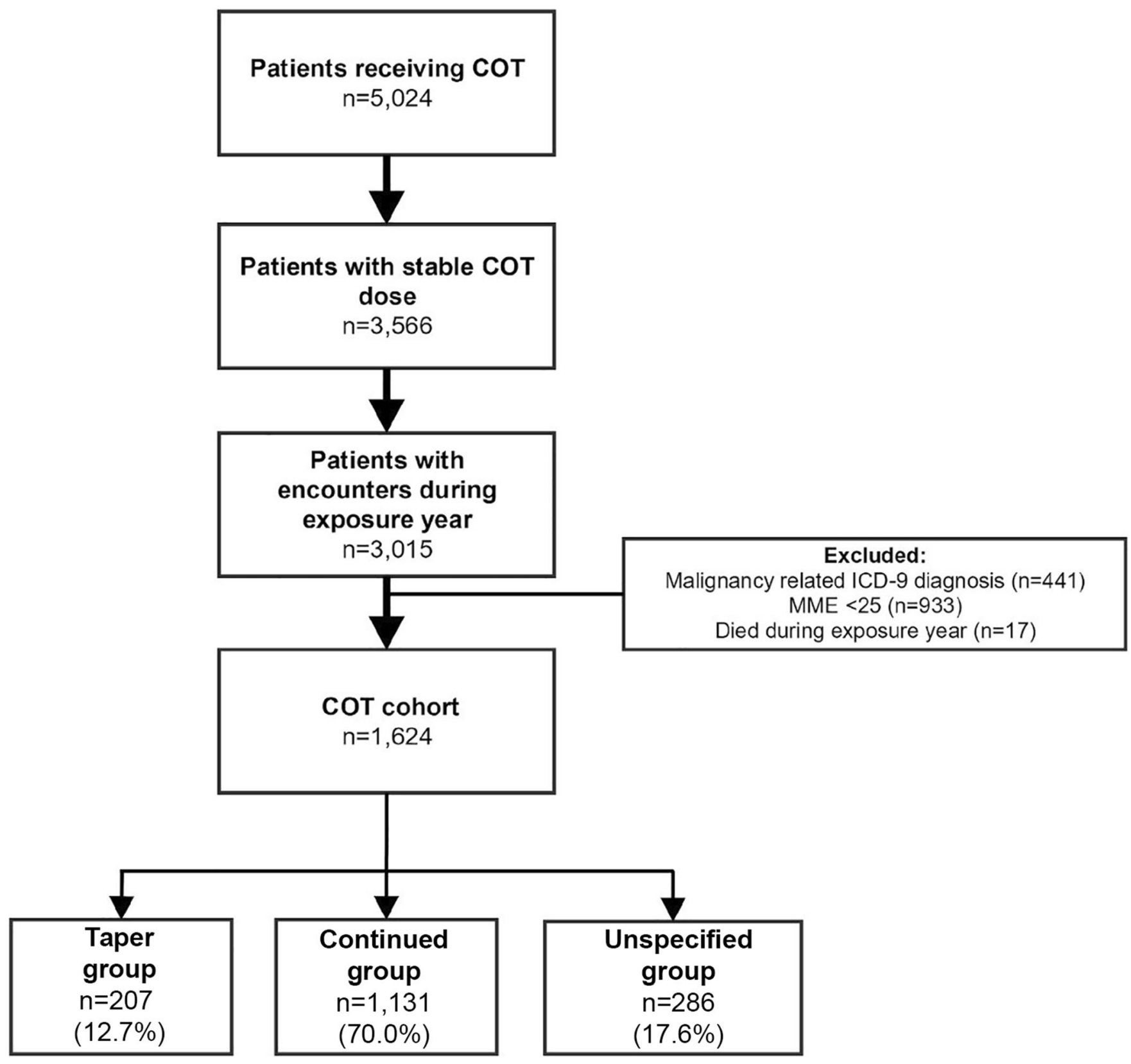

Figure 2 Consort flow diagram.

odds of termination of care (AOR 4.3 [95\% CI 2.2-8.5]) (Table 2). A post hoc power analysis showed our primary analysis with a sample size of 1338 was well powered (> $90 \%$ ) to detect the estimated AOR of 4.3 .

Findings remained significant in sensitivity analyses. In the first sensitivity analysis comparing patients with opioid tapers versus those who continued or were unspecified, opioid taper was associated with two times greater odds of termination of care (AOR 2.2 [95\% CI 1.2-3.8]). In the second sensitivity analysis comparing the taper group and the unspecified group to the continued group, opioid taper was associated with greater than six times greater odds of termination of care (AOR 6.3 [95\% CI $3.8-10.7])$.
Using a 50\% taper definition, $131(8.1 \%)$ patients had an opioid taper, 1299 (79.9\%) had dose continuation, and 194 (12.0\%) were unspecified. Among the 78 patients who experienced termination of care, $12(9.2 \%)$ had experienced a taper, $32(2.5 \%)$ had been continued, and $34(17.5 \%)$ were unspecified. In our sensitivity analysis comparing patients with $50 \%$ tapers versus those continued on opioids, opioid taper was associated with almost four times the odds of termination of care (AOR 3.8 [95\% CI 1.8-7.9]). Additional sensitivity analyses considering different approaches to the unspecified group that utilized a $50 \%$ taper definition were similar in magnitude and direction to those that utilized a $30 \%$ taper definition and remained significant, with adjusted odds ranging from 2.1 to 6.4 (see Online Appendix Table 3). 
Table 1 Participant Baseline Characteristics $(n=1624)$

\begin{tabular}{ll}
\hline \hline Characteristic & $\boldsymbol{n}(\%)$ \\
\hline Age, mean (SD) & $51.5(11.7)$ \\
Gender, $n(\%)$ & \\
-Female & $881(54.3)$ \\
-Male & $743(45.8)$ \\
Race/ethnicity, $n(\%)$ & \\
-White, non-Hispanic & $267(16.4)$ \\
-Black/African-American, non-Hispanic & $542(33.4)$ \\
-Hispanic/Latino & $507(31.2)$ \\
-Other (including multiracial) or unknown & $308(19.0)$ \\
Language & $1408(86.7)$ \\
-English & $206(12.7)$ \\
-Spanish & $10(0.6)$ \\
-Other & $90(46.7-193.1)$ \\
Baseline opioid dose, median MME (IQR) & \\
Pain type at baseline, $n(\%)^{*}$ & $1025(63.1)$ \\
-Back pain & $1028(63.3)$ \\
-Extremity pain & $151(9.3)$ \\
-Neck pain & $334(20.6)$ \\
-Headache pain & $431(26.5)$ \\
-Neuropathic pain & $472(29.1)$ \\
-Abdominal pain & \\
Diagnoses at baseline, $n(\%)$ & $324(20.0)$ \\
-Non-opioid drug use disorder & $101(6.2)$ \\
-Alcohol use disorder & $423(26.1)$ \\
-Tobacco use disorder & $767(47.2)$ \\
-Mental health disorder & ${ }^{\dagger}$ \\
-Hypertension & $817(50.3)$ \\
-Diabetes & $358(22.0)$ \\
\hline
\end{tabular}

*Based on provider ICD-9 coding; categorizations based on approach of Larochelle et al. Patients may have multiple pain diagnoses

Based on ICD-9 coding; includes codes for anxiety, depression, bipolar disorder, PTSD, and schizophrenia

\section{CONCLUSIONS}

In this retrospective study of patients on a stable opioid dose for chronic pain between 2008 and 2012 with follow-up through 2014, an opioid taper was associated with greater than four times the odds of termination of care in the subsequent year when compared with opioid dose continuation. In sensitivity analyses, results remained qualitatively consistent, with

Table 2 Association of Opioid Tapering and Termination of Care

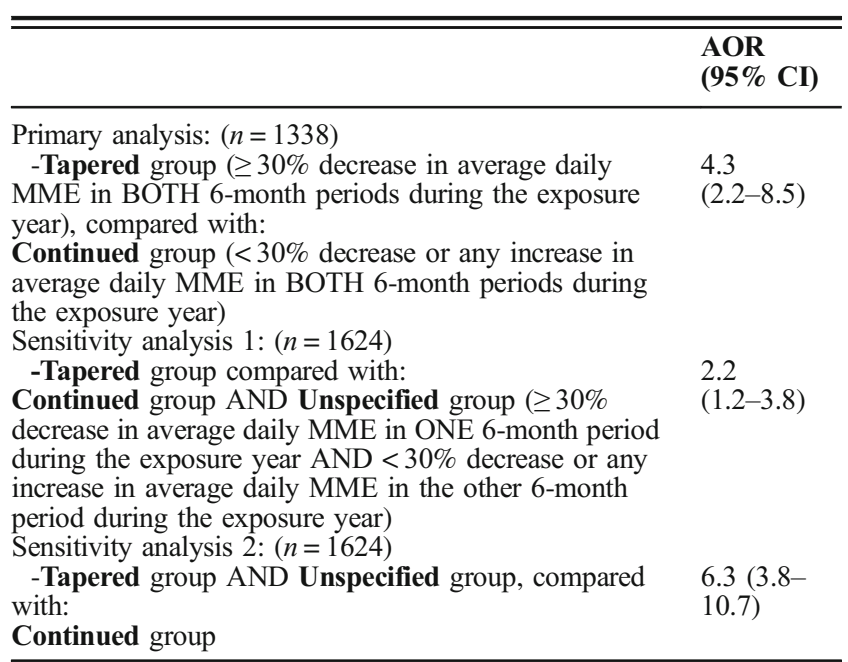

All models adjusted for patient age, gender, race and ethnicity, preferred language (English vs. non-English speaking), baseline opioid dose, total outpatient visits during exposure year, Elixhauser medical complexity score, the number of pain category diagnoses, and the patient's baseline year a range of 2.1 to 6.4 fold increased odds of termination of care. This study is the first to examine the relationship between an opioid taper and subsequent termination of care within a health system and adds to the nascent literature about outcomes of opioid tapering in real-world settings, where tapers are becoming more commonplace. 4

These findings raise concern about the potential for unintended consequences of opioid tapering. Termination of care, either patient or provider initiated, has risks and can lead to poor patient outcomes if a patient obtains care in a new and different setting or falls out of care entirely. Termination of care has been linked to poor health outcomes for chronic diseases such as asthma, diabetes, and HIV. ${ }^{1-13,22}$ Patients with substance use disorder who drop out of treatment programs have higher unemployment, incarceration, and relapse rates. ${ }^{10}$ Patients undergoing an opioid taper who fall out of care may be at risk for opioid misuse or overdose. They may have difficulty finding a new provider who is willing to prescribe opioids to them. ${ }^{23,24}$ Such patients, often physically dependent on opioids and experiencing distress from opioid withdrawal symptoms, may seek other sources of opioids, including emergency rooms or illegal and illicit sources. ${ }^{25}$ Additionally, illicit opioid use puts individuals at risk for overdose and death, given the current prevalence of fentanyl and fentanyl metabolites found in illicit opioids. $^{26,27}$ Other consequences that have not been directly studied could include initiation of injection drug use with resultant risks of HIV or hepatitis C transmission. ${ }^{28}$ Depression and suicidality associated with poorly controlled chronic pain are another concern. ${ }^{29,} 30$ Findings from this study suggest that patients who taper may be at risks for negative clinical outcomes due to their increased risk of terminating care.

Nonetheless, many of the risks of opioid tapering are still theoretical, as evidence to date is lacking. For example, studies linking opioid tapering to opioid overdoses or mortality are inconclusive. Among 11 poor-quality studies of opioid tapering that reported mortality outcomes reviewed for a recent systematic review, one overdose-related death was reported from a total participant pool of 519. ${ }^{16}$ Owing to the rarity of such important outcomes, further large-scale studies are needed to supplement the evidence base to more definitively guide clinicians in weighing the true risks and benefits of opioid tapering.

Evidence suggests that tapering can be associated with improvements in pain and function, but this may not reflect the realities of involuntary tapers in real-world settings. ${ }^{16-18}$ Involuntary tapering is indicated in certain circumstances for patients for whom risks clearly exceed benefits of continuing, such as after a non-fatal overdose event. ${ }^{2,3,31}$ Nonetheless, in response to the current opioid overdose crisis in the USA, providers may feel external pressure to make unilateral decisions about tapering in circumstances where the risks do not clearly exceed the benefits. For example, the quantity and dose of opioid prescriptions may soon appear in a provider's quality metrics and be directly tied to financial compensation. ${ }^{32,33}$ The results of the current study should invite caution and demonstrate the need to fully understand the risks and benefits of opioid tapers. 
This study has several limitations. First, it was conducted in a single health system using data from 2008 to 2014 which may limit generalizability to other settings. It is possible results may differ in the present, given the rapidly shifting landscape of opioid prescribing and tapering. Second, we could not determine the intent behind an opioid taper or whether termination in care was a direct result of an opioid taper. Third, our definition of opioid taper was conservative, requiring dose reductions in two consecutive 6-month periods. Our findings left a substantial group classified as "unspecified." Notably, this group had the highest rates of terminating their care, but this may have reflected, among other reasons, termination of care late in the exposure year rather than a taper. Because we were unable to determine whether they had tapered, the unspecified group was only used in sensitivity analyses to provide ranges for our effect estimates. Fourth, our definition of termination of care was conservative, requiring no outpatient encounters with any provider in the health system in the outcome year. By our definition, patients who ended their care with their main prescriber to switch to a different provider within our health system would not be classified as having terminated their care.

Patients in a large urban medical center who had opioid tapers were more likely to experience subsequent termination of care compared with patients taking chronic opioid therapy who had dose continuation. In light of widespread adoption of recommendations to taper select patients, these findings should invite caution. As current opioid tapering guidelines indicate, ${ }^{2,3,31}$ providers should carefully consider the risks of opioid tapering against the benefits of opioid tapering, especially if considering a unilateral decision to taper opioids without patient agreement or engagement. Additional research is needed to more definitively characteristic risks and benefits of opioid tapering in real-world settings and to identify best practices for engaging patients in an opioid taper effectively and for retaining them in care.

Corresponding Author: Hector R. Perez, MD, MS; Montefiore Medical Center Albert Einstein College of Medicine, Bronx, NY, USA (e-mail: heperez@montefiore.org).

Funding Information Financial support for this project was provided by the following grants: K23DA044327 (PI: Perez), RO1DA039046 and K24DA046309 (PI: Starrels), and K24DA036955 (PI: Cunningham).

\section{Compliance with Ethical Standards:}

The study was approved by the Albert Einstein College of Medicine and Montefiore Medical Center Institutional Review Board.

Conflict of Interest: Dr. Starrels discloses receiving research support from the Opioid Post-Marketing Requirement Consortium, an FDAmandated consortium of opioid manufacturers, for an observational study of the risks of opioids. Dr. Cunningham discloses holding stock and stock options in Quest Diagnostics. All remaining authors declare that they do not have conflict of interests.

\section{REFERENCES}

1. Berna C, Kulich RJ, Rathmell JP. Tapering Long-term Opioid Therapy in Chronic Noncancer Pain. Mayo Clin Proc. 2015;90(6):828-842.

2. Dowell D, Haegerich TM, Chou R. CDC. Guideline for Prescribing Opioids for Chronic Pain - United States, 2016. Morb Mortal Wkly Rep. 2016;65(1).

3. The Management of Opioid Therapy for Chronic Pain Working Group. VA/DoD Clinical Practice Guideline for Management of Opioid Therapy for Chronic Pain. Department of Veterans Affairs, Department of Defense; 2010.

4. Dowell D, Haegerich TM. Changing the Conversation About Opioid Tapering. Ann Intern Med. 2017;167(3):208-209.

5. Pezalla EJ, Rosen D, Erensen JG, Haddox JD, Mayne TJ. Secular trends in opioid prescribing in the USA. J Pain Res. 2017;10:383-387.

6. Kennedy LC, Binswanger IA, Mueller SR, et al. "Those Conversations in My Experience Don't Go Well": A Qualitative Study of Primary Care Provider Experiences Tapering Long-term Opioid Medications. Pain Med. 2018;19(11):2201-2211.

7. Hawkins EJ, Malte CA, Hagedorn HJ, et al. Survey of Primary Care and Mental Health Prescribers' Perspectives on Reducing Opioid and Benzodiazepine Co-Prescribing Among Veterans. Pain Med. 2017;18(3):454467.

8. Matthias MS, Johnson NL, Shields CG, et al. "I'm Not Gonna Pull the Rug out From Under You": Patient-Provider Communication About Opioid Tapering. J Pain. 2017;18(11):1365-1373.

9. Frank JW, Levy C, Matlock DD, et al. Patients' Perspectives on Tapering of Chronic Opioid Therapy: A Qualitative Study. Pain Med. 2017;17(10):1838-1847.

10. Stark MJ. Dropping out of substance abuse treatment: A clinically oriented review. Clin Psychol Rev. 1992;12(1):93-116.

11. McGovern CM, Redmond M, Arcoleo K, Stukus DR. A missed primary care appointment correlates with a subsequent emergency department visit among children with asthma. J Asthma. 2017;54(9):977-982.

12. Schectman JM, Schorling JB, Voss JD. Appointment adherence and disparities in outcomes among patients with diabetes. J Gen Intern Med. 2008;23(10): 1685-1687.

13. Harrison LB, Lingvay I. Appointment and medication non-adherence is associated with increased mortality in insulin-treated type 2 diabetes. BMJ Evid. Based Med. 2013;18(3):112-113.

14. Buonora M, Perez HR, Heo M, Cunningham CO, Starrels JL. Race and Gender Are Associated with Opioid Dose Reduction Among Patients on Chronic Opioid Therapy [published online ahead of print Jul 18, 2018]. Pain Med.

15. Starrels JL, Becker WC, Weiner MG, Li X, Heo M, Turner BJ. Low use of opioid risk reduction strategies in primary care even for high risk patients with chronic pain. J Gen Intern Med. 2011;26(9):958-964.

16. Frank JW, Lovejoy TI, Becker WC, et al. Patient Outcomes in Dose Reduction or Discontinuation of Long-Term Opioid Therapy: A Systematic Review. Ann Intern Med. 2017;167(3):181-191.

17. Darnall BD, Ziadni MS, Stieg RL, Mackey IG, Kao M-C, Flood P. Patient-Centered Prescription Opioid Tapering in Community Outpatients With Chronic Pain. JAMA Intern Med. 2018;178(5):707-708.

18. Sullivan MD, Turner JA, DiLodovico C, D'Appollonio A, Stephens K, Chan Y-F. Prescription Opioid Taper Support for Outpatients With Chronic Pain: A Randomized Controlled Trial. J Pain. 2017;18(3):308318.

19. Larochelle MR, Liebschutz JM, Zhang F, Ross-Degnan D, Wharam JF. Opioid Prescribing After Nonfatal Overdose and Association With Repeated Overdose: A Cohort Study. Ann Intern Med. 2016;164(1):1-9.

20. Smith SM, Dart RC, Katz NP, et al. Classification and definition of misuse, abuse, and related events in clinical trials: ACTTION systematic review and recommendations. Pain. 2013;154(11):2287-2296.

21. Guan $\mathbf{H}$, Sundararajan V, Halfon $\mathbf{P}$, et al. Coding algorithms for defining comorbidities in ICD-9-CM and ICD-10 administrative data. Med Care. 2005;43(11):1130-1139.

22. Hall BJ, Sou K-L, Beanland $\mathbf{R}$, et al. Barriers and Facilitators to Interventions Improving Retention in HIV Care: A Qualitative Evidence Meta-Synthesis. AIDS Behav. 2017;21(6):1755-1767.

23. Fuqua A. The other opioid crisis: pain patients who can't access the medicine we need. The Washington Post. https://www.washingtonpost. com/outlook/the-other-opioid-crisis-pain-patients-who-cant-access-themedicine-they-need/2018/03/09/5ad83b24-2301-11e8-badd7c9f29a55815_story.html?utm_term=.1a24c9ele84f. Published March 9, 2018. Accessed 8 May 2019. 
24. Masters K. Chronic pain patients report struggles under tighter opioid regulations. The Frederick News-Post. https://www.fredericknewspost. com/news/health/hospitals_and_doctors/chronic-pain-patients-reportstruggles-under-tighter-opioid-regulations/article_f6cdf517-ba94-59a68395-644ecccc77e2.html. Published February 12, 2018. Accessed 8 May 2019.

25. Han B, Compton WM, Blanco C, Crane E, Lee J, Jones CM. Prescription Opioid Use, Misuse, and Use Disorders in U.S. Adults: 2015 National Survey on Drug Use and Health. Ann Intern Med. 2017;167(5):293-301.

26. Carroll JJ, Marshall BDL, Rich JD, Green TC. Exposure to fentanylcontaminated heroin and overdose risk among illicit opioid users in Rhode Island: A mixed methods study. Int J Drug Policy. 2017;46:136145.

27. Macmadu A, Carroll JJ, Hadland SE, Green TC, Marshall BDL. Prevalence and correlates of fentanyl-contaminated heroin exposure among young adults who use prescription opioids non-medically. Addict Behav. 2017;68:35-38.

28. Muhuri PK, Gfroerer JC, Davies MC. Associations of Nonmedical Pain Reliever Use and Initiation of Heroin Use in the United States. Substance Abuse and Mental Health Services Administration, CBHSQ Data Review. August 2013. http://www.samhsa.gov/data/sites/default/files/DR006/ DR006/nonmedical-pain-reliever-use-2013.htm. Accessed 8 May 2019.

29. Ilgen MA, Bohnert ASB, Ganoczy D, Bair MJ, McCarthy JF, Blow FC. Opioid dose and risk of suicide. Pain. 2016;157(5):1079-1084.

30. Oquendo MA, Volkow ND. Suicide: A Silent Contributor to OpioidOverdose Deaths. N Engl J Med. 2018;378(17):1567-1569.
31. Washington State Medical Directors Group. Interagency Guideline on Prescribing Opioids for Pain. Vol 3rd. Agency Medical Directors' Group; 2015. http://www.agencymeddirectors.wa.gov/Files / 2015AMDGOpioidGuideline.pdf. Accessed 8 May 2019.

32. NQF Statement on Endorsement of Opioid Patient Safety Measures. National Quality Forum. https://www.qualityforum.org/News_And_Resources/Press_Releases/2017/NQF_Statement_on_Endorsement_of_ Opioid_Patient_Safety_Measures.aspx. Accessed 8 May 2019.

33. NCQA Updates Quality Measures for HEDIS 2019. National Committee for Quality Assurance. https://www.ncqa.org/news/ncqa-updates-quality-measures-for-hedis-2019/. Accessed 8 May 2019.

Publisher's Note Springer Nature remains neutral with regard to jurisdictional claims in published maps and institutional affiliations.

\section{APPENDIX}

Table 3 Association of Opioid Tapering (50\% Taper) and Termination of Care

\begin{tabular}{|c|c|}
\hline & $\begin{array}{l}\text { AOR } \\
(95 \% \mathrm{CI})\end{array}$ \\
\hline \multicolumn{2}{|l|}{$50 \%$ sensitivity analysis: $(n=1430)$} \\
\hline$-\mathbf{5 0} \%$ Tapered group $(\geq 50 \%$ decrease in average daily & 3.8 \\
\hline $\begin{array}{l}\text { MME in BOTH } 6 \text { month periods during the exposure } \\
\text { year), compared with: }\end{array}$ & $(1.8-7.9)$ \\
\hline \multicolumn{2}{|l|}{$\begin{array}{l}\text { Continued group }(<50 \% \text { decrease or any increase in } \\
\text { average daily MME in BOTH } 6 \text { month periods during } \\
\text { the exposure year })\end{array}$} \\
\hline \multicolumn{2}{|l|}{ Sensitivity analysis $1:(n=1624)$} \\
\hline$-\mathbf{5 0 \%}$ Tapered group compared with: & 2.1 \\
\hline $\begin{array}{l}50 \% \text { Continued group AND } 50 \% \text { Unspecified group } \\
(\geq 50 \% \text { decrease in average daily } \mathrm{MME} \text { in } \mathrm{ONE}\end{array}$ & $(1.1-4.2)$ \\
\hline \multirow{2}{*}{\multicolumn{2}{|c|}{$\begin{array}{l}6 \text {-month period during the exposure year AND }<50 \% \\
\text { decrease or any increase in average daily MME in the } \\
\text { other } 6 \text {-month period during the exposure year) }\end{array}$}} \\
\hline & \\
\hline \\
\hline$-\mathbf{5 0 \%}$ Tapered group AND $\mathbf{5 0 \%}$ Unspecified group, & $6.4(4.0-$ \\
\hline compared with: & $10.5)$ \\
\hline $50 \%$ Continued group & \\
\hline
\end{tabular}

